\title{
Genetic alteration of stemness factors and p53 in mouse forestomach by chemical carcinogen-induced carcinogenesis
}

\author{
KAZUYOSHI YAMAMOTO, HIDESHI ISHII, JEONG HO MOON, \\ NAOTSUGU HARAGUCHI, YUICHIRO DOKI and MASAKI MORI
}

\begin{abstract}
Department of Gastroenterological Surgery, Graduate School of Medicine, Osaka University, Suita, Osaka 565-0871, Japan
\end{abstract}
Received December 16, 2011; Accepted February 17, 2012

DOI: $10.3892 / \mathrm{ol} .2012 .642$

\begin{abstract}
The expression profiles of genes normally enriched in embryonic stem (ES) cells (stemness factors) are associated with poor clinical outcome in solid tumors. However, whether such gene expression is responsible for tumor initiation and progression remains to be determined. The tumor suppressor gene p53 is known to attenuate the expression of Nanog, which is essential for maintaining stem cells in response to DNA damage. On the basis of these findings, we hypothesized that stemness factors and p53 closely correlate with each other and form a network in response to genomic damage in the early phase of carcinogenesis and in the process of tumor progression. In this study, we applied the N-methylbenzylnitrosoamine (NMBA)-induced carcinogenesis model to the mouse forestomach to clarify the role of the stemness factors, c-Myc, Klf4, Sox2, Oct3/4 and Nanog, in cancer development using p53(+/+) $(n=26)$ and $\mathrm{p} 53(+/-)(\mathrm{n}=11) \mathrm{C} 57 \mathrm{BL} / 6 \mathrm{~J}$ mice. Thirty weeks following NMBA administration, histologically evident squamous cell carcinoma was detected in the forestomachs of p53(+/+) mice, and the percentage of p53-positive nuclei in the forestomach epithelium gradually increased during carcinogenesis. Tumor development in p53(+/-) mice occurred significantly earlier than in p53(+/+) mice. Quantitative real-time PCR analyses revealed a reduced c-Myc and Klf4 expression before evident morphological changes were observed, and an increased expression with the development of squamous cell carcinoma. Sox 2 expression remained unchanged until tumor development and increased with the appearance of squamous cell carcinomas. The expression of Oct $3 / 4$ and Nanog increased at the early stages following NMBA administration, and Nanog expression in situ was not positively affected by the deficiency of p53. Findings of the present study suggested that Oct3/4
\end{abstract}

Correspondence to: Dr Masaki Mori, Department of Gastroenterological Surgery, Graduate School of Medicine, Osaka University, 2-2, E2, Yamadaoka, Suita, Osaka 565-0871, Japan E-mail: mmori@gesurg.med.osaka-u.ac.jp

Key words: carcinogenesis, stemness factors, forestomach, squamous cell carcinoma may be involved in the progression of carcinogenesis from normal epithelial cells at early stages, suggesting the potential use of Oct3/4 as a biomarker in forestomach tumor formation at early stages of chemical carcinogenesis.

\section{Introduction}

Reprogramming of somatic cells into induced pluripotent stem cells was achieved by gene transfection of 4 transcription factors, c-Myc, Sox 2, Oct3/4, and Klf4 (1-3). These 4 transcription factors and Nanog are overexpressed in embryonic stem (ES) cells, known to be regulators of identity of ES cells, and are responsible for the maintenance of ES cells. Recent attention has focused on functions of these genes, i.e., how they act as stemness factors. Besides reprogramming to pluripotent cells and maintaining their pluripotency and self-renewing nature, there has been increasing evidence in terms of such stemness factors as involved in malignancies. Microarray analysis by Ben-Porath et al showed that aggressive breast cancer preferentially overexpressed genes normally enriched in ES cells, and this ES-like genetic signature in breast cancer is associated with poor clinical outcome (4). It is known that c-Myc is an oncogene, Sox 2 expression is involved in invasion and metastasis of pancreatic intraepithelial neoplasia (5), Oct $3 / 4$ expression is involved in tumorigenesis via the activation of its downstream genes in breast cancer-initiating cells (6), upregulation of Klf4 in esophageal epithelial cells induces inflammation-mediated esophageal squamous cell cancer $(7,8)$, and Nanog is expressed in various types of tumor, including carcinoma of the breast, cervix and ovary (9-11). On the basis of these findings, stemness factors may contribute to carcinogenesis, invasion and cancer metastasis.

The tumor suppressor gene p53 is a signifcant negative regulator of carcinogenesis (12). It is activated in response to DNA damage stress signals and then binds to the promoter of Nanog and suppresses Nanog expression $(13,14)$. Cancer development involves the accumulation of genetic changes that lead to malignant transformation of normal cells $(15,16)$. It is supposed that stemness factors and p53 may form a network and regulate the downstream gene expression during carcinogenesis prior to morphological changes (17). If the early genetic alteration of such genes in cancer development were revealed, it would be possible to achieve the early detection of malignancy and optimal oncological therapy, and also 
implement a risk evaluation of precancerous lesions prior to cancer development, enabling the rapid application of chemoprevention therapies targeted at these genes.

However, there have been few reports showing such genetic alterations in cancer development, particularly in the early stage of cancer or in the case of precancerous lesions (18). This lack of evidence may be due to the difficulty in modeling longitudinal genetic alterations in the normal epithelial-dysplasia carcinoma sequence in humans (19). To examine this notion, we used the carcinogen-induced carcinogenesis model of mice, which uses $\mathrm{N}$-methylbenzylnitrosoamine (NMBA) administration to induce squamous cell carcinoma in the mouse forestomach.

\section{Materials and methods}

Mice. C57BL/6J mice p53+/+ (CLEA Japan, Inc., Tokyo, Japan) were crossed with C57BL/6J p53+/- mice (RIKEN Japan, Saitama, Japan). The p53 offspring were differentiated by the genotyping of tail DNA by quantitative real-time PCR (qPCR). The animals were maintained in hanging polycarbonate cages and were provided with food, at a controlled temperature $\left(23 \pm 1^{\circ} \mathrm{C}\right)$ and a 12 -h light/dark cycle, in accordance with the NIH Guide for Care and Use of Laboratory Animals.

Chemical carcinogen-induced carcinogenesis. The animal studies were approved by the Institutional Animal Care and Use Committee at Osaka University, Japan. Six-week-old p53+/+ mice $(n=26)$ and $p 53+/-$ mice $(n=11)$ were administered 6 intraesophageal doses of NMBA ( $2 \mathrm{mg} / \mathrm{kg}$ weight) twice weekly, via a thin tube over 3 weeks (20); the NMBA caused squamous cell carcinoma in the mouse forestomach. P53(+/+) and p53(+/-) C57BL/6J mice ( $n=5$ each; age, 26 weeks) not treated with NMBA were used for evaluating sporadic forestomach tumor development. P53+/+ mice were sacrificed at weeks $4(n=5)$, $8(n=5), 12(n=5), 20(n=6)$ and $30(n=5)$ following NMBA administration, and p53 +/- mice were sacrificed at weeks $4(n=4), 8(n=2), 12(n=3)$ and $20(n=2)$ following NMBA administration. The mice were analyzed for tumor incidence by longitudinally opening the stomach and counting the number of mice-bearing tumors in the forestomach. The opened stomach was divided into 2 sections: one section was fixed in $10 \%$ buffered formalin, embedded in paraffin, and stained with H\&E for histopathology or immunohistochemistry (IHC), and the other section was used for RNA extraction for performing qPCR and microarray analyses.

IHC. IHC was performed using the Histofine Mousestain kit. Paraffin-embedded sections $(5 \mu \mathrm{m})$ were deparaffinized in xylene and dehydrated through graded ethanol. The sections were immersed in antigen retrieval buffer ( $\mathrm{pH}$ 6.0, citrate buffer) and heated for $10 \mathrm{~min}$ at $121^{\circ} \mathrm{C}$ in an autoclave. Continuous sections were incubated with $3 \% \mathrm{H}_{2} \mathrm{O}_{2}$ to block endogenous peroxidase activity. Slides were then incubated with goat serum for $20 \mathrm{~min}$ to reduce non-specific background staining. Blocked sections were incubated at a 1:50 dilution of a primary antibody (rabbit polyclonal anti-p53 antibody; SC-6243; Santa Cruz Biotechnology Inc., Santa Cruz, CA, USA) and incubated overnight at $4^{\circ} \mathrm{C}$. The subsequent reaction was performed using the EnVision kit (Dako, Carpinteria, CA, USA) according to the manufacturer's instructions, followed by DAB development. The slides were then counterstained with hematoxylin. IHC analysis was performed by evaluating the percentage of p53-positive nuclei in the mouse forestomach epithelium.

$q P C R$. Total RNA was extracted from the forestomach of the sacrificed mice using TRIzol reagent (Invitrogen Life Technologies, Carlsbad, CA, USA). Reverse transcription was performed using a SuperScript III reverse transcription kit (Invitrogen). qPCR was performed using the LightCycler TaqMan Master kit (Roche Diagnostics, Tokyo) for cDNA amplification of specific target genes. Purified cDNA from mouse ES cells was used as a positive control for the target genes. The expression of mRNA copies was normalized to GAPDH mRNA expression. Primers for Oct3/4, c-Myc, Klf-4, Sox 2 and Nanog were used.

Statistical analysis. Continuous values were expressed as the mean \pm standard error. The correlation between the target gene expression and tumor incidence differences was analyzed by the $\chi^{2}$ test or Fisher's exact test. Statistical analyses were performed using JMP v8.0 software (SAS Institute, Cary, NC, USA). $\mathrm{P}<0.05$ was considered to indicate a statistically significant difference.

\section{Results}

NMBA-induced carcinogenesis. A total of 37 mice (26 C57BL/6J p53+/+ and 11 C57BL/6J p53+/-) were administered intraesophagus doses of NMBA. C57BL/6J p53+/+ mice were sacrificed at weeks $4(n=5), 8(n=5), 12(n=5), 20(n=6)$ and $30(n=5)$ following NMBA administration, and C57BL/6J p53+/-mice were sacrificed at weeks $4(n=4), 8(n=2)$, $12(n=3)$ and $20(n=2)$ following NMBA administration. Macroscopic findings revealed that in the p53+/+ mice, despite evident changes not being observed until 12 weeks after NMBA administration, elevated tumors and thickened forestomach were detected in 2 of 6 mice 20 weeks following NMBA administration and in 5 of 5 mice 30 weeks following NMBA administration. Thirty weeks after NMBA administration, histological examination revealed an evident squamous cell carcinoma in the forestomach that had metastasized into submucosal layer. In the p53(+/-) mice, elevated tumors and thickened forestomachs were already evident in 2 of 4 mice at 4 weeks, in 2 of 2 mice at 8 weeks, in 3 of 3 mice at 12 weeks and in 2 of 2 mice at 20 weeks following NMBA administration. In the p53(+/+) and p53(+/-) mice not treated with $\operatorname{NMBA}(\mathrm{n}=5$ in each; age, 26 weeks), there was no evidence of sporadic forestomach tumors (Fig. 1).

p53 IHC. IHC analysis of p53 was performed for each p53(+/+) mouse tissue sample, and the percentage of p53-positive nuclei in the forestomach epithelium was determined. In the control mice, p53 was rarely expressed in the forestomach epithelium, with only $12.6 \%$ p53-positive nuclei. The expression of p53 gradually increased at 4, 8, 12, 20 and 30 weeks following NMBA administration. Notably, the percentage of p53positive nuclei at 30 weeks following NMBA administration was $66.2 \%$, and p53 was overexpressed in the squamous cell carcinoma lesions (Fig. 2). 

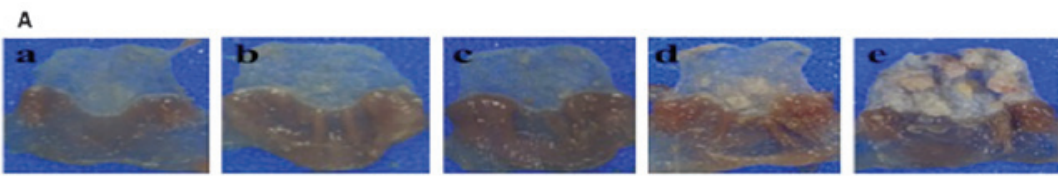

B
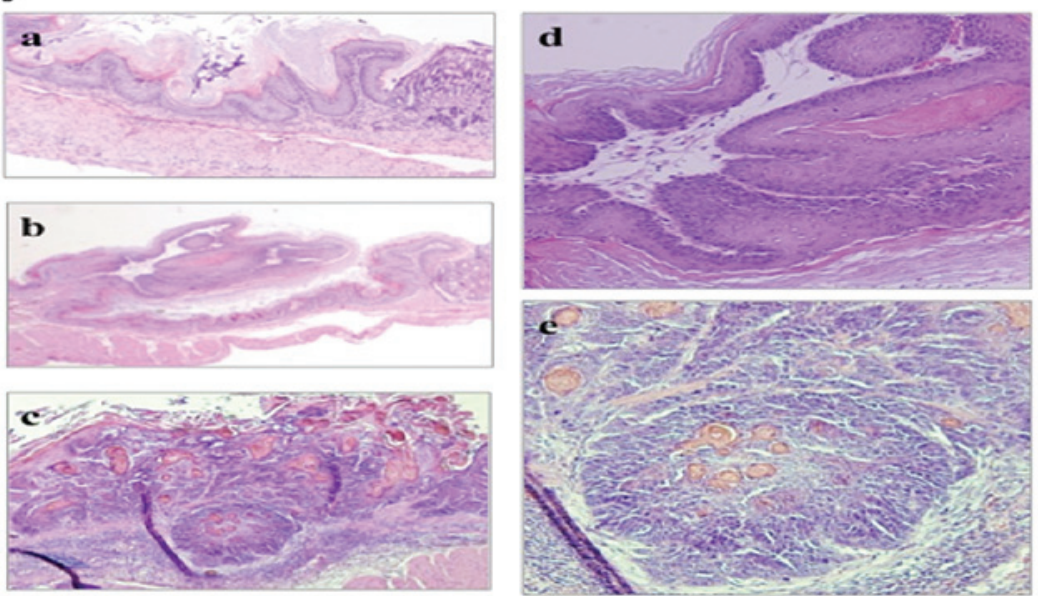

Figure 1. Macroscopic and histological appearance in C57BL/6J p53+/+ forestomach following NMBA administration. A: Macroscopic appearance. A total of (a) 4 weeks, (b) 8 weeks, (c) 12 weeks, (d) 20 weeks and (e) 30 weeks following NMBA treatment. Thickened forestomach and elevated tumors were detected from 20 weeks following NMBA administration. B: Histological appearance of H\&E-stained sections. (a) Normal forestomach epithelium without NMBA treatment. (b and d) Twenty weeks following NMBA administration, the sections demonstrated intraepithelial hyperplasia. (c and e) Thirty weeks following NMBA administration, there was an evident squamous cell carcinoma histologically in the forestomach that had invaded into the submucosal layer.

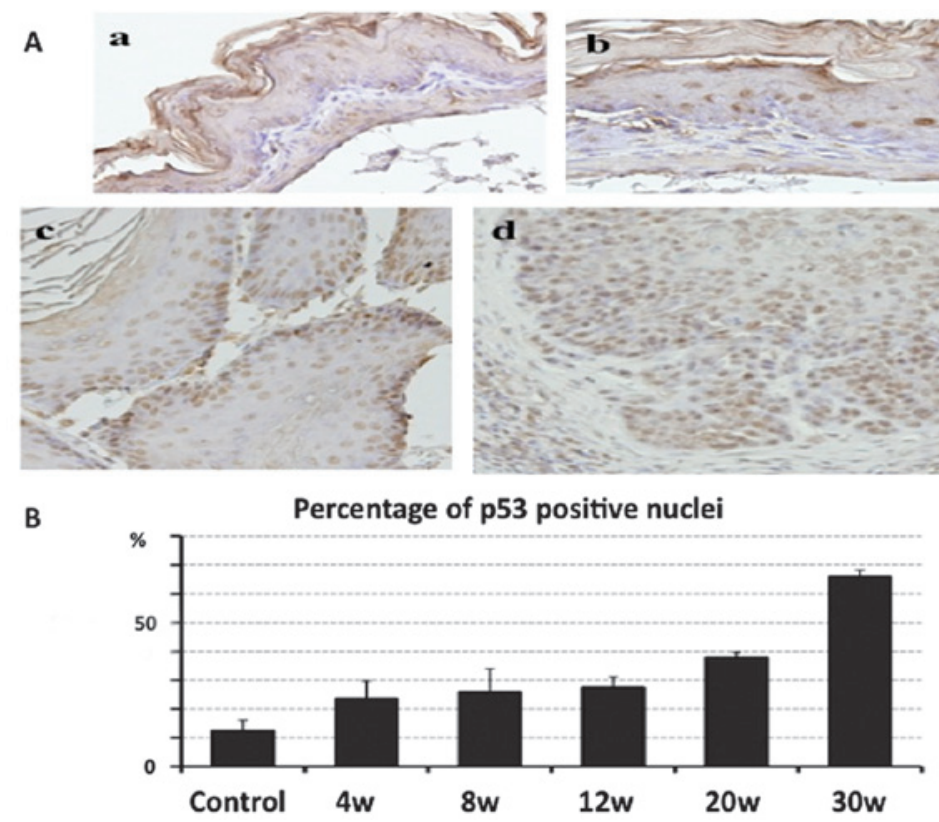

Figure 2. Immunohistochemical staining of p53 in C57BL/6J p53+/+ forestomach following NMBA administration. A: (a) P53 staining of normal mice forestomach without NMBA treatment. A total of (b) 4 weeks, (c) 12 weeks and (d) 30 weeks following NMBA administration is shown. B: The percentage of p53-positive nucleus in the forestomach epithelium. The p53 expression was gradually increased, and in 30 weeks following NMBA administration in mice, p53 was overexpressed in squamous cell carcinoma lesions; $\mathrm{n}=5$ for each group.

Expression pattern of stemness factors. Bulk RNA was extracted from the forestomachs of the sacrificed mice and qPCR analysis of stemness factors relative to GAPDH was performed. It was predicted that the expression of the stemness factors in the p53+/+ mice would be upregulated in the early phase of carcinogenesis. The qPCR analysis demonstrated that the c-Myc and Klf4 expression decreased at 12 and 20 weeks following administration of the treatment (before evident morphological changes were observed), and increased at 30 weeks following administration. The Sox 2 expression in the mouse ES cell line (positive control) and in mice 20 weeks following NMBA administration were compared and a significant increase was observed at 30 weeks following NMBA administration. There was little Oct $3 / 4$ and Nanog expression 
c-Myc
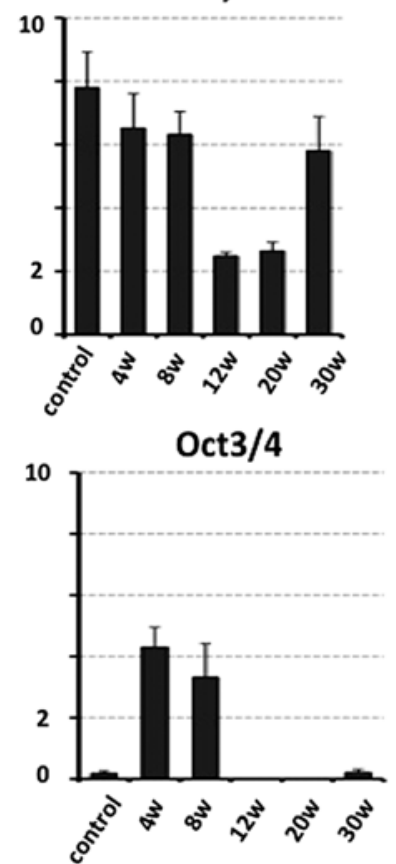

KIf4
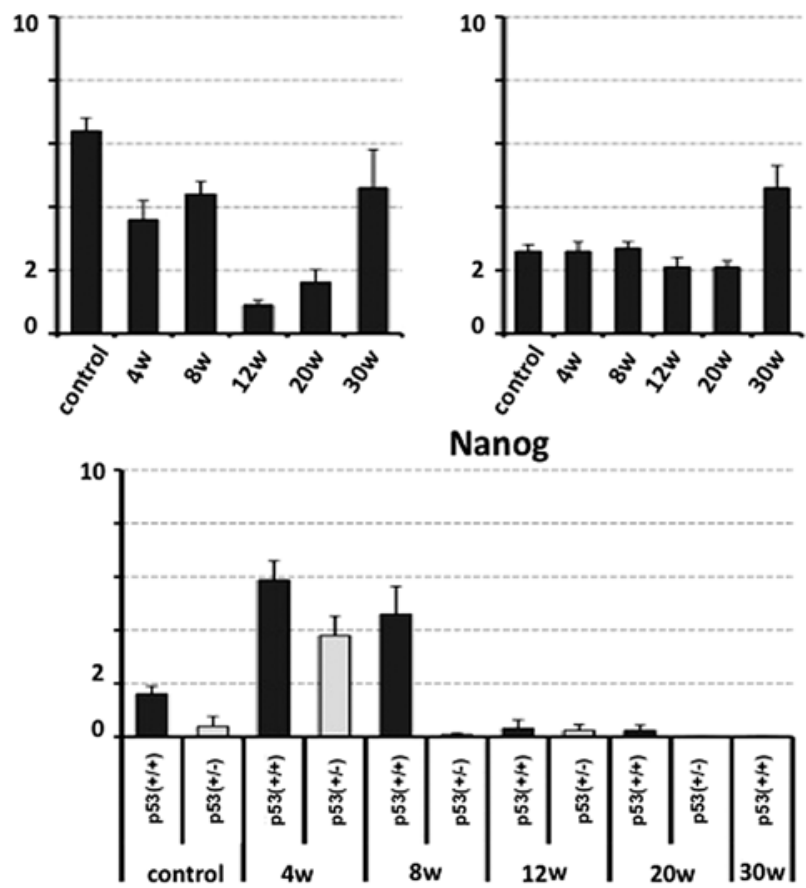

Figure 3. Quantitative real-time PCR analysis of stemness factors (c-Myc, K1f4, Sox 2 and Oct3/4) relative to GAPDH were performed by using the bulk RNA extracted from the C57BL/6J p53+/+ forestomach. PCR analysis of Nanog was specifically conducted by using the C57BL/6J p53+/+ and C57BL/6J p53+/forestomach; $\mathrm{n}=5$ for each group.

in control tissue samples compared with the positive control for qPCR detection (Fig. 3). The expression of Oct3/4 and Nanog increased at the early stages following NMBA administration, and Nanog expression was not positively affected by the deficiency of p53.

\section{Discussion}

This study was designed to examine the genetic alteration of stemness factors in the early initiation phase of carcinogenesis using chemical carcinogen-induced carcinogenesis in vivo. NMBA has been widely used to induce tumors in the rodent esophagus and forestomach by producing an electrophilic methylating agent that produces the mutagenic adduct $\mathrm{O}^{6}$-methylguanine in DNA $(21,22)$. Previous studies that examined the gene expression associated with cancer were mainly horizontal comparison analyses that compared normal epithelia to cancer lesions or multiple surgically resected specimens. Few studies, however, have reported longitudinal or serial changes during carcinogenesis $(23,24)$. A plausible carcinogenesis model may make significant assessments possible before evident morphological changes have been established.

We hypothesized that the early detection of increased gene expression in stemness factors in several types of cancer, where stemness factors are overexpressed compared with those in the normal epithelium, is useful in the detection of cancer in highrisk groups, or for adopting interventional therapy to prevent cancer development (chemoprevention). Nanog and Oct3/4 were expressed in the early stages of carcinogenesis, and the expression of c-Myc and Klf4 was decreased once during the precancerous stage and increased when apparent tumors containing squamous cell carcinoma developed. The Sox2 expression level remained unchanged before evident tumors appeared, but significantly increased when tumors evidently developed. Furthermore, we examined the correlation between Nanog and p53 using p53(+/-) mice; however, the unexpectedly heterozygous deletion of p53 did not upregulate Nanog expression.

In the present study, we investigated the molecular events, i.e., any alterations of expression of immature-related genes, including c-Myc, Klf4, Nanog, Oct3/4 and Sox 2 (reprogramming factors), before apparent tumors were established in the mouse forestomach. The data demonstrated that, although further study is required for Nanog, the expression of Oct3/4 was increased even in 'normal' epithelial cells, suggesting that Oct $3 / 4$ is involved in the progression of carcinogenesis from normal epithelial cells at early stages. Thus, the study indicates a use for this gene as a biomarker in forestomach tumor formation.

\section{Acknowledgements}

This study was partly supported by a grant from the Core Research for Evolutional Science and Technology (CREST) (H.I., N.H., M.M.), a Grant-in-Aid for Scientific Research on Priority Areas (D.Y., M.M.), a Grant-in-Aid for Scientific Research from the Ministry of Education, Culture, Sports, Science and Technology (H.I., D.Y., M.M.), a Grant-in-Aid for the 3rd Comprehensive 10-year Strategy for Cancer Control Ministry of Health, Labour and Welfare (H.I., M.M.), a grant from the Tokyo Biochemical Research Foundation (M.M.) and a grant from the Princess Takamatsu Cancer Research Fund, Japan (H.I.). 


\section{References}

1. Takahashi K and Yamanaka S: Induction of pluripotent stem cells from mouse embryonic and adult fibroblast cultures by defined factors. Cell 126: 663-676, 2006.

2. Takahashi K, Tanabe K, Ohnuki M, Narita M, Ichisaka T, Tomoda $\mathrm{K}$ and Yamanaka S: Induction of pluripotent stem cells from adult human fibroblasts by defined factors. Cell 131: 861-872, 2007.

3. Yamanaka S: Elite and stochastic models for induced pluripotent stem cell generation. Nature 460: 49-52, 2009.

4. Ben-Porath I, Thomson MW, Carey VJ, Ge R, Bell GW, Regev A and Weinberg RA: An embryonic stem cell-like gene expression signature in poorly differentiated aggressive human tumors. Nat Genet 40: 499-507, 2008.

5. Lengerke C, Fehm T, Kurth R, Neubauer H, Scheble V, Müller F, Schneider F, Petersen K, Wallwiener D, Kanz L, Fend F, Perner S, Bareiss PM and Staebler A: Expression of the embryonic stem cell marker SOX2 in early-stage breast carcinoma. BMC Cancer 11: 42, 2011

6. Ponti D, Costa A, Zaffaroni N, Pratesi G, Petrangolini G, Coradini D, Pilotti S, Pierotti MA and Daidone MG: Isolation and in vitro propagation of tumorigenic breast cancer cells with stem/progenitor cell properties. Cancer Res 65: 5506-5511, 2005.

7. Tetreault MP, Wang ML, Yang Y, Travis J, Yu QC, Klein-Szanto AJ and Katz JP: Klf4 overexpression activates epithelial cytokines and inflammation-mediated esophageal squamous cell cancer in mice. Gastroenterology 139: 2124-2134, 2010.

8. Tian Y, Luo A, Cai Y, Su Q, Ding F, Chen H, and Liu Z: MicroRNA-10b promotes migration and invasion through KLF4 in human esophageal cancer cell lines. J Biol Chem 285: 7986-7994, 2010.

9. Jeter CR, Badeaux M, Choy G, Chandra D, Patrawala L, Liu C, Calhoun-Davis T, Zaehres H, Daley GQ and Tang DG: Functional evidence that the self-renewal gene NANOG regulates human tumor development. Stem Cells 27: 993-1005, 2009.

10. Glinsky GV: 'Stemness' genomics law governs clinical behavior of human cancer: implications for decision making in disease management. J Clin Oncol 26: 2846-2853, 2008.

11. Chiou SH, Wang ML, Chou YT, Chen CJ, Hong CF, Hsieh WJ, Chang HT, Chen YS, Lin TW, Hsu HS and Wu CW: Coexpression of Oct4 and Nanog enhances malignancy in lung adenocarcinoma by inducing cancer stem cell-like properties and epithelial-mesenchymal transdifferentiation. Cancer Res 70 10433-10444, 2010.
12. Hickman ES, Moroni MC and Helin K: The role of p53 and pRB in apoptosis and cancer. Curr Opin Genet Dev 12: 60-66, 2002.

13. Pan G and Thomson JA: Nanog and transcriptional networks in embryonic stem cell pluripotency. Cell Res 17: 42-49, 2007.

14. Lin T, Chao C, Saito S, Mazur SJ, Murphy ME, Appella E and $\mathrm{Xu}$ Y: p53 induces differentiation of mouse embryonic stem cells by suppressing Nanog expression. Nat Cell Biol 7: 165-171, 2005.

15. Bartkova J, Horejsi Z, Koed K, Kramer A, Tort F, Zieger K, Guldberg P, Sehested M, Nesland JM, Lukas C, Orntoft T, Lukas J and Bartek J: DNA damage response as a candidate anti-cancer barrier in early human tumorigenesis. Nature 434: 864-870, 2005

16. Gorgoulis VG, Vassiliou LV, Karakaidos P,Zacharatos P, Kotsinas A, Liloglou T, Venere M, Ditullio RA Jr, Kastrinakis NG, Levy B, Kletsas D, Yoneta A, Herlyn M, Kittas C and Halazonetis TD: Activation of the DNA damage checkpoint and genomic instability in human precancerous lesions. Nature 434: 907-913, 2005

17. Rich AM, Kerdpon D and Reade PC: p53 expression in oral precancer and cancer. Aust Dent J 44: 103-105, 1999.

18. Pontén J: Cell biology of precancer. Eur J Cancer 37: S97-S113, 2001.

19. Ozols R: Esophageal cancer. Curr Problems Cancer 18: 191-246, 1994.

20. Fong LYY, Ishii H, Nguyen VT, Vecchione A, Farber JT, Croce CM and Huebner K: p53 deficiency accelerates induction and progression of esophageal and forestomach tumors in zinc-deficient mice. Cancer Res 63: 186-195, 2003.

21. Carlton PS, Kresty LA, Siglin JC, Morse MA, Lu J, Morgan C and Stoner GD: Inhibition of N-nitrosomethylbenzylamine-induced tumorigenesis in the rat esophagus by dietary freeze-dried strawberries. Carcinogenesis 22: 441-446, 2001.

22. Dumon KR, Ishii H, Fong LYY, Zanesi N, Fidanza V, Mancini R, Vecchione A, Baffa R, Trapasso F, During MJ, Huebner K and Croce CM: FHIT gene therapy prevents tumor development in Fhit-deficient mice. Proc Natl Acad Sci USA 98: 3346-3351, 2001.

23. Coia LR: The esophagus. In: Moss' Radiation Oncology: Rationale, Techniques, Results. Cox J (ed.) Mosby-Year Book, St. Louis, p409, 1994.

24. Blot WJ: Esophageal cancer trends and risk factors. Semin Oncol 21: 403-410, 1994. 